\title{
Interaction of Solitary Waves in the System Described by the Kadomtsev-Petviashvili Equation with a Self-Consistent Source
}

\section{V.K. Mel'nikov}

Laboratory of Theoretical Physics, Joint Institute for Nuclear Research, Head Post Office P.O. Box 79, SU-101000 Moscow, USSR

\begin{abstract}
Solitary waves moving with nonconstant velocity are found in the nonlinear integrable system described by the Kadomtsev-Petviashvili equation with a self-consistent source. Explicit expressions are derived for the solutions describing the interaction of an arbitrary number of these waves. It is shown that in contrast with the decay and fusion of solitons, the decay and fusion of the above solitary waves are not of the resonance nature and proceed in the general case. The obtained results are relevant to some problems of hydrodynamics, solid state physics, plasma physics, etc.
\end{abstract}

The goal of the present paper is the interaction of solitary waves in the system described by the equations $[1-4]$

$$
3 \frac{\partial^{2} u}{\partial y^{2}}-\frac{\partial}{\partial x}\left[\frac{\partial u}{\partial t}+\frac{\partial}{\partial x}\left(3 u^{2}+\frac{\partial^{2} u}{\partial x^{2}}+8 \kappa|\varphi|^{2}\right)\right]=0, \quad i \frac{\partial \varphi}{\partial y}=u \varphi+\frac{\partial^{2} \varphi}{\partial x^{2}} .
$$

This system arises in studying the interaction of long and short waves in different physical processes; here, respectively, $u$ is the long wave amplitude, $\varphi$ is the complex envelope of a short wave packet, and the parameter $\kappa$ satisfies the condition $\kappa^{2}=1$

As is known, the system (1) has a one-soliton solution of the form

$$
\begin{aligned}
& u=\frac{2 \mu^{2}}{\cosh ^{2}[\mu(x+2 v y-\tau t)+\delta]}, \\
& \varphi=a \frac{\exp \left[i v x-i\left(\mu^{2}-v^{2}\right) y+i \sigma t\right]}{\cosh [\mu(x+2 v y-\tau t)+\delta]},
\end{aligned}
$$

where the real parameters $\mu, v$, and $\tau$ and the complex quantity $a$ satisfy the only condition

$$
4 \kappa|a|^{2}=\left[\tau-4\left(\mu^{2}-3 v^{2}\right)\right] \mu^{2},
$$

and the quantities $\delta$ and $\sigma$ take any real values. It follows from this relation that for the solitons (2) to exist the parameters $\mu, v$, and $\tau$ should satisfy the inequality

$$
\left[\tau-4\left(\mu^{2}-3 v^{2}\right)\right] \kappa \geqq 0 .
$$


The parameters $\delta$ and $\theta=\arg a$ characterize the position of the solution (2) on the $x, y$ plane, and at $\mu^{2}+v^{2}>0$ by the change $x \rightarrow x+x_{0}$ and $y \rightarrow y+y_{0}$ they can be transformed into any a priori given quantities, for instance, equal to zero. By using four real parameters $\mu, v, \sigma$, and $\tau$ we form two complex quantities $\omega$ and $\varrho$ of the form

$$
\omega=\mu+i v, \varrho^{3}=-\bar{\omega}^{3}+\frac{1}{4}(\mu \tau+i \sigma)
$$

playing an important role in studying the interaction of solitons (2). Hereafter the bar means complex conjugation.

It follows from relation (3) that at $\tau=4\left(\mu^{2}-3 v^{2}\right)$ the soliton (2) degenerates into the well known soliton [5]

$$
u=\frac{2 \mu^{2}}{\cosh ^{2}\left[\mu(x+2 v y)-4 \mu\left(\mu^{2}-3 v^{2}\right) t+\delta\right]}, \varphi \equiv 0
$$

of the Kadomtsev-Petviashvili equation [6]. According to (5) the parameter $\varrho^{3}$ can take only pure imaginary values. Then, assuming $\sigma=\tau=0$, according to (5) we find that $\varrho^{3}=-\bar{\omega}^{3}$. In this case the soliton (2) takes the form

$$
\begin{aligned}
& u=\frac{2 \mu^{2}}{\cosh ^{2}[\mu(x+2 v y)+\delta]}, \\
& \varphi=a \frac{\exp \left[i v x-i\left(\mu^{2}-v^{2}\right) y\right]}{\cosh [\mu(x+2 v y)+\delta]},
\end{aligned}
$$

i.e., is the stationary solution of the system (1). Consequently, the soliton (7) satisfies the system of equations

$$
\begin{gathered}
3 \frac{\partial^{2} u}{\partial y^{2}}-\frac{\partial^{2}}{\partial x^{2}}\left(3 u^{2}+\frac{\partial^{2} u}{\partial x^{2}}+8 \kappa|\varphi|^{2}\right)=0 \\
i \frac{\partial \varphi}{\varphi y}=u \varphi+\frac{\partial^{2} \varphi}{\partial x^{2}}
\end{gathered}
$$

following from the system (1) after neglecting the term $\frac{\partial u}{\partial t}$ in the first equation. Finally, note that $N>1$ solitons (2) with equal quantities $\omega_{m}$ but with different quantities $\varrho_{m}^{3}, m=1, \ldots, N$, form a solitary wave of the form

$$
\begin{aligned}
u & =\frac{2 \mu^{2}}{\cosh ^{2}[\mu(x+2 v y-f)]}, \\
\varphi & =A \frac{\exp \left[i v x-i\left(\mu^{2}-v^{2}\right) y\right]}{\cosh [\mu(x+2 v y-f)]},
\end{aligned}
$$

where the quantities $f$ and $A$ are independent of the coordinates $x$ and $y$. However, these quantities depend on the time $t$ and satisfy the relation

$$
\frac{d f}{d t}=4\left(\mu^{2}-3 v^{2}\right)+4 \kappa \mu^{-2}|A|^{2} \text {. }
$$

It follows from this relation that $\frac{d^{2} f}{d t^{2}} \neq 0$ if at this moment of time the inequality $\frac{d|A|^{2}}{d t} \neq 0$ holds. Thus, the change in the square of the $\varphi$-wave amplitude leads to a 
synchronous acceleration of the $u$-wave and vice versa, acceleration of the $u$-wave leads to a synchronous change of the square of the $\varphi$-wave amplitude.

One can easily be convinced that relation (10) is a necessary and sufficient condition for the functions $u$ and $\varphi$ determined by (9) to satisfy the system (1). Moreover, as will be shown below, the class of the pairs of functions $f, A$ satisfying relation (10) is essentially wider than the one that can be obtained from the $N$-soliton solution of the system (1) under the above choice of the parameters $\omega_{m}$ and $\varrho_{m}^{3}, m=1, \ldots, N$. Thus, waves of the form (9) are not always obtained from the multi-soliton solution of the system (1). Nevertheless, the interaction of $N>1$ waves of the form (9) is always described by the formulae very similar in structure with those for the $N$-soliton solution of the system (1). Below, we shall derive these formulae for investigating the interaction of solitary waves (9). In particular, we will show that under certain conditions two or more waves (9) can be in a bound state, i.e., during a semi-infinite interval of time $t<0$, these waves are at finite distance from each other, then the bound state decays and for $t>0$ these waves move away from each other at an infinite distance. Then, if in these solutions one changes $t$ by $-t$ and $x$ by $-x$ the new solutions of the system (1) will describe the process of forming a bound state, i.e., the process in which two or more solitary waves (9) for $t<0$ are at infinite distance from each other and for all $t>0$ will be at finite distance from each other. It should be noted that in contrast with the decay and fusion of solitons being as is known of the resonance nature, the decay and formation of a bound state of the waves (9) proceed under the conditions given by the inequalities, i.e., in the general case.

\section{1. $N$-Wave Solution of the System (1)}

To derive formulae describing the interaction $N>1$ of the waves (9) we take a vector-column $\lambda$ with $N$ components $\lambda_{m}$ of the form

$$
\lambda_{m}=\exp \left(\omega_{m} x-i \omega_{m}^{2} y\right), m=1, \ldots, N,
$$

where $\omega_{m}$ are complex parameters. Then, we take the square matrix $P$ of an order of $N$ with elements $P_{m, n}$ of the form

$$
P_{m, n}=\frac{\lambda_{m} \bar{\lambda}_{n}}{\omega_{m}+\bar{\omega}_{n}}, m, n=1, \ldots, N .
$$

Let finally $q$ be a vector-column with $N$ components $q_{1}, \ldots, q_{N}$, and $Q$ be a Hermitian matrix of an order of $N$ with elements $Q_{m, n}, m, n=1, \ldots, N$, such that the equality

$$
\frac{d Q}{d t}+4 \bar{\omega}^{3} Q+4 Q \omega^{3}+4 \kappa \bar{q} \tilde{q}=0
$$

holds where

$$
\omega=\operatorname{diag}\left(\omega_{1}, \ldots, \omega_{N}\right),
$$

and the sign " $"$ means transposition, i.e., in particular, transition from the vector-column to the vector-row. Thus, the expression $\bar{q} \tilde{q}$ defines the Hermitian matrix of an order of $N$ with the elements $\bar{q}_{m} q_{n}, m, n=1, \ldots, N$. Assume now that 
the components $q_{m}$ of the vector $q$ and the elements $Q_{m, n}$ of the matrix $Q$ are independent of the coordinates $x$ and $y$. We put

$$
D=\operatorname{det}\left|\begin{array}{cc}
\mathbb{1} & -Q \\
P & \mathbb{1}
\end{array}\right|, \Phi=\operatorname{det}\left|\begin{array}{ccc}
0 & 0 & \tilde{q} \\
0 & \mathbb{1} & -Q \\
\lambda & P & \mathbb{1}
\end{array}\right|,
$$

where $\mathbb{1}$ is the unit matrix of the order $N$. Then, the functions

$$
u=2 \frac{\partial^{2}}{\partial x^{2}} \ln D, \quad \varphi=\frac{\Phi}{D}
$$

satisfy the system (1), where $D \neq 0$.

Taking account of (1.6) we easily find that this assertion can be proved by verifying the relations

$$
\begin{gathered}
\left(3 \frac{\partial^{2} D}{\partial y^{2}}-\frac{\partial^{2} D}{\partial t \partial x}-\frac{\partial^{4} D}{\partial x^{4}}\right) D-3\left[\left(\frac{\partial^{2} D}{\partial x^{2}}\right)^{2}+\left(\frac{\partial D}{\partial y}\right)^{2}\right] \\
+\left(\frac{\partial D}{\partial t}+4 \frac{\partial^{3} D}{\partial x^{3}}\right) \frac{\partial D}{\partial x}-4 \kappa|\Phi|^{2}=0 \\
\left(i \frac{\partial \Phi}{\partial y}-\frac{\partial^{2} \Phi}{\partial x^{2}}\right) D=\left(i \frac{\partial D}{\partial y}+\frac{\partial^{2} D}{\partial x^{2}}\right) \Phi-2 \frac{\partial D}{\partial x} \frac{\partial \Phi}{\partial x} .
\end{gathered}
$$

The validity of these relations, as will be shown below, can be proved by the following algebraic lemma.

Algebraic Lemma. Let $B$ be the square matrix of an order of $r+2, r>0$. Let then $B_{\mu, v}$ be the square matrix of an order of $r+1$ obtained from the matrix $B$ after cancelling the elements of the $\mu^{\text {th }}$ row and $v^{\text {th }}$ column, and $\beta_{\mu, v}=\operatorname{det} B_{\mu, v}$, $\mu, v=1, \ldots, r+2$. Let finally $B_{0}$ be a minor of the $r^{\text {th }}$ order in the right lower angle of the matrix $B$. Then, the equality

$$
(\operatorname{det} B)\left(\operatorname{det} B_{0}\right)=\beta_{1,1} \beta_{2,2}-\beta_{1,2} \beta_{2,1}
$$

is valid.

The proof of this lemma is trivial, and therefore, we omit it (see, for instance, paper [3]).

Let us show now how the relations (1.7) and (1.8) follow from equality (1.9). For this purpose we use the square matrices $F_{m, n}, G_{m}, U$ and $V$ of the form

$$
\begin{gathered}
F_{m, n}=\left|\begin{array}{ccc}
0 & \lambda^{*} \bar{\omega}^{n} & 0 \\
0 & \mathbb{1} & -Q \\
\omega^{m} \lambda & P & \mathbb{1}
\end{array}\right| m \geqq 0, n \geqq 0, \\
G_{m}=\left|\begin{array}{ccc}
0 & 0 & \tilde{q} \\
0 & \mathbb{1} & -Q \\
\omega^{m} \lambda & P & \mathbb{1}
\end{array}\right| m \geqq 0,
\end{gathered}
$$




$$
\begin{aligned}
& U=\left|\begin{array}{cccr}
0 & 0 & \lambda^{*} \bar{\omega} & 0 \\
0 & 0 & \lambda^{*} & 0 \\
0 & 0 & \mathbb{1} & -Q \\
\omega \lambda & \lambda & P & \mathbb{1}
\end{array}\right|, \\
& V=\left|\begin{array}{cccc}
0 & 0 & 0 & \tilde{q} \\
0 & 0 & \lambda^{*} & 0 \\
0 & 0 & \mathbb{1} & -Q \\
\omega \lambda & \lambda & P & \mathbb{1}
\end{array}\right| .
\end{aligned}
$$

Hereafter the asterisk will mean the Hermitian conjugation, i.e., transposition and complex conjugation performed simultaneously. One can easily see that the matrices $F_{m, n}$ and $G_{m}$ are of the order $2 N+1$ and the matrices $U$ and $V$ are of the order $2 N+2$. By simple calculations based on equalities (1.1), (1.2), and (1.5) one can verify the relations

$$
\begin{aligned}
& \frac{\partial D}{\partial x}=-\operatorname{det} F_{0,0}, \quad \frac{\partial^{2} D}{\partial x^{2}}=-\operatorname{det} F_{0,1}-\operatorname{det} F_{1,0}, \\
& \frac{\partial^{3} D}{\partial x^{3}}=-\operatorname{det} F_{0,2}-2 \operatorname{det} F_{1,1}-\operatorname{det} F_{2,0}, \\
& \frac{\partial^{4} D}{\partial x^{4}}=-\operatorname{det} F_{0,3}-3 \operatorname{det} F_{1,2}-3 \operatorname{det} F_{2,1}-\operatorname{det} F_{3,0}+2 \operatorname{det} U, \\
& \frac{\partial \Phi}{\partial x}=\operatorname{det} G_{1}, \frac{\partial^{2} \Phi}{\partial x^{2}}=\operatorname{det} G_{2}-\operatorname{det} V, \\
& \frac{\partial D}{\partial y}=-i \operatorname{det} F_{0,1}+i \operatorname{det} F_{1,0}, \\
& \frac{\partial^{2} D}{\partial y^{2}}=\operatorname{det} F_{0,3}-\operatorname{det} F_{1,2}-\operatorname{det} F_{2,1}+\operatorname{det} F_{3,0}-2 \operatorname{det} U, \\
& \frac{\partial \Phi}{\partial y}=-i \operatorname{det} G_{2}-i \operatorname{det} V .
\end{aligned}
$$

Hence it follows that

$$
\begin{aligned}
& i \frac{\partial D}{\partial y}+\frac{\partial^{2} D}{\partial x^{2}}=-2 \operatorname{det} F_{1,0}, \\
& i \frac{\partial \Phi}{\partial y}-\frac{\partial^{2} \Phi}{\partial x^{2}}=2 \operatorname{det} V .
\end{aligned}
$$

Let us use the algebraic lemma. Assume $B=V$. Then, according to (1.10), (1.11), and (1.13) we have

$$
B_{1,1}=F_{0,0}, B_{1,2}=F_{1,0}, B_{2,1}=G_{0}, B_{2,2}=G_{1} .
$$

Then, based on (1.13) we get the equality

$$
B_{0}=\left|\begin{array}{rr}
\mathbb{1} & -Q \\
P & \mathbb{1}
\end{array}\right| .
$$


By virtue of (1.5) it follows that

$$
\operatorname{det} B_{0}=D
$$

Thus, in this case equality (1.9) takes the form

$$
D \operatorname{det} V=\operatorname{det}\left|\begin{array}{ll}
\operatorname{det} F_{0,0} & \operatorname{det} G_{0} \\
\operatorname{det} F_{1,0} & \operatorname{det} G_{1}
\end{array}\right|
$$

According to equalities (1.5), (1.11), (1.14), (1.15), and (1.17) we get that relation (1.18) coincides with (1.8). Consequently, the validity of relation (1.8) is proved. Now we calculate the quantity $\frac{\partial D}{\partial t}$. For this purpose we use the diagonal
matrix $T$ of the form

$$
T=\exp \left(4 \omega^{3} t\right)
$$

where the matrix $\omega$ is determined by equality (1.4), and assume

$$
\hat{P}=T^{-1} P \bar{T}^{-1}, \hat{Q}=\bar{T} Q T .
$$

Based on (1.3) the matrix $\hat{Q}$ satisfies the condition

$$
\frac{d \hat{Q}}{d t}+4 \kappa \bar{T} \bar{q} \tilde{q} T=0
$$

On the other hand, by virtue of (1.5) we have

$$
D=\operatorname{det}\left|\begin{array}{rr}
\mathbb{1} & -\hat{Q} \\
\hat{P} & \mathbb{1}
\end{array}\right| \text {. }
$$

Taking account of (1.1), (1.2), (1.10), and (1.19)-(1.21) we get the equality

$$
\frac{\partial D}{\partial t}=4 \operatorname{det} F_{0,2}-4 \operatorname{det} F_{1,1}+4 \operatorname{det} F_{2,0}-4 \kappa \operatorname{det} R
$$

where

$$
R=\left|\begin{array}{rrr}
0 & 0 & \tilde{q} \\
\bar{q} & \mathbb{1} & -Q \\
0 & P & \mathbb{1}
\end{array}\right|
$$

Then, the equality

$$
\frac{\partial^{2} D}{\partial t \partial x}=4 \operatorname{det} F_{0,3}+4 \operatorname{det} F_{3,0}+4 \operatorname{det} U+4 \kappa \operatorname{det} W
$$

is valid where

$$
W=\left|\begin{array}{rrrr}
0 & 0 & 0 & \tilde{q} \\
0 & 0 & \lambda^{*} & 0 \\
\bar{q} & 0 & \mathbb{1} & -Q \\
0 & \lambda & P & \mathbb{1}
\end{array}\right| .
$$


Thus, according to (1.14), (1.16), (1.22), and (1.24) we get the equality

$$
\begin{aligned}
& 3 \frac{\partial^{2} D}{\partial y^{2}}-\frac{\partial^{2} D}{\partial t \partial x}-\frac{\partial^{4} D}{\partial x^{4}}=-12 \operatorname{det} U-4 \kappa \operatorname{det} W \\
& \frac{\partial D}{\partial t}+4 \frac{\partial^{3} \mathrm{D}}{\partial \mathrm{x}^{3}}=-12 \operatorname{det} F_{1,1}-4 \kappa \operatorname{det} R \\
& \left(\frac{\partial^{2} D}{\partial x^{2}}\right)^{2}+\left(\frac{\partial D}{\partial y}\right)^{2}=4\left(\operatorname{det} F_{0,1}\right)\left(\operatorname{det} F_{1,0}\right) .
\end{aligned}
$$

Let us use the algebraic lemma again. Let $B=U$. Then, according to equalities (1.10) and (1.12) we get that

$$
B_{1,1}=F_{0,0}, B_{1,2}=F_{1,0}, \quad B_{2,1}=F_{0,1}, \quad B_{2,2}=F_{1,1} .
$$

Then, based on (1.5) and (1.12) we have the equality det $B_{0}=D$. Thus, in the case considered relation (1.9) becomes

$$
D \operatorname{det} U=\operatorname{det}\left|\begin{array}{ll}
\operatorname{det} F_{0,0} & \operatorname{det} F_{0,1} \\
\operatorname{det} F_{1,0} & \operatorname{det} F_{1,1}
\end{array}\right| \text {. }
$$

Let then $B=W$. Taking account of (1.5), (1.10), (1.23) and (1.25) we get that

$$
B_{1,1}=F_{0,0}, \quad \operatorname{det} B_{2,1}=\Phi, \quad B_{2,2}=R, \operatorname{det} B_{0}=D .
$$

Then, based on the matrices $P$ and $Q$ being Hermitian we can verify the equality

$$
\bar{\Phi}=\operatorname{det}\left|\begin{array}{rrr}
0 & \lambda^{*} & 0 \\
\bar{q} & \mathbb{1} & -Q \\
0 & P & \mathbb{1}
\end{array}\right|,
$$

i.e., by virtue of (1.25) we have $\operatorname{det} B_{1,2}=\bar{\Phi}$. Consequently, relation (1.9) now becomes

$$
D \operatorname{det} W=\operatorname{det}\left|\begin{array}{cc}
\operatorname{det} F_{0,0} & \bar{\Phi} \\
\Phi & \operatorname{det} R
\end{array}\right| .
$$

According to equalities (1.26) validity of relation (1.7) is proved by relations (1.27) and (1.28). Thus, the validity of relations (1.7) and (1.8) is proved.

It should be mentioned that the Hermitian matrix $P$ determined by (1.1) and (1.2) will be non-negative if the quantities $\omega_{m}$ satisfy the condition

$$
\operatorname{Re} \omega_{m}>0, \quad m=1, \ldots, N \text {. }
$$

On the contrary, the matrix $P$ will be nonpositive if the condition

$$
\text { Re } \omega_{m}<0, \quad m=1, \ldots, N,
$$

is fulfilled. Thus, choosing the Hermitian matrix $Q$ non-negative under the condition (1.29) or nonpositive if the condition (1.30) holds, in both the cases taking account of (1.5) we are convinced in the validity of the inequality $D \geqq 1$. This means that the solution of the system (1) determined by (1.6) has no singularities in this case. Moreover, from the matrices $P$ and $Q$ being Hermitian it follows that the function $u$ determined by (1.5) and (1.6) takes only real values at any real values of $x, y$ and $t$. 
Let now

$$
\begin{aligned}
q_{m} & =a_{m} \exp \left[-4\left(\omega_{m}^{3}+\bar{\varrho}_{m}^{3}\right) t\right], \quad m=1, \ldots, N, \\
Q_{m, n} & =\kappa \frac{\bar{q}_{m} q_{n 12}}{\varrho_{m}^{3}+\bar{\varrho}_{n}^{3}}, \quad m, n=1, \ldots, N,
\end{aligned}
$$

where $a_{m} \neq 0, \omega_{m}$ and $\varrho_{m}^{3}$ are the complex parameters. By simple calculations we get that in this case the vector $q$ and Hermitian matrix $Q$ satisfy equality (1.3). According to (5) we assume

$$
\omega_{m}=\mu_{m}+i v_{m}, \varrho_{m}^{3}=-\bar{\omega}_{m}^{3}+\frac{1}{4}\left(\mu_{m} \tau_{m}+i \sigma_{m}\right), \quad m=1, \ldots, N,
$$

where $\mu_{m}, v_{m}, \sigma_{m}$ and $\tau_{m}$ are the real parameters. One can easily be convinced that the matrix $Q$ will be non-negative if the condition (1.29) is fulfilled, and the parameters $\mu_{m}, v_{m}$ and $\tau_{m}$ satisfy an analogous to (4) inequality

$$
\left[\tau_{m}-4\left(\mu_{m}^{2}-3 v_{m}^{2}\right)\right] \kappa>0, \quad m=1, \ldots, N
$$

On the contrary, the matrix $Q$ will be nonpositive if the conditions (1.30) and (1.32) are fulfilled simultaneously. Consequently, choosing the quantities $\omega_{m}$ and $\varrho_{m}^{3}$ either according to conditions (1.29) and (1.32) or to (1.30) and (1.32), we get the solution of the system (1) having no singularities at any real values of $x, y$ and $t$. The solution of the system (1) thus obtained is the known $N$-soliton solution [3]. It describes the interaction of $N$ solitons of the form (2). If in this solution at some values of the index $m$ we put $\tau_{m}=4\left(\mu_{m}^{2}-3 v_{m}^{2}\right)+\kappa\left|a_{m}\right|^{2} c_{m}$, where $c_{m}>0$, and pass to the limit as $a_{m} \rightarrow 0$, then the obtained $N$-soliton solution of the system (1) will contain a number of solitons of the form (6). Then, assuming $\varrho_{m}^{3}=-\bar{\omega}_{m}^{3}$ at some values of the index $m$ we succeed in that our $N$-soliton solution of the system (1) will contain also a number of solitons of the form (7). Finally, putting in the initial solution $\varrho_{m}^{3}=-\bar{\omega}_{m}^{3}$ at all values of the index $m=1, \ldots, N$ we get $N$-soliton solution of the system (8).

Now in equalities (1.31) we put $\omega_{1}=\ldots=\omega_{N}=\mu+i v$, where $\mu$ and $v$ are the real parameters. By simple calculations we can verify the equalities

$$
\begin{aligned}
& D=1+K \exp [2 \mu(x+2 v y)], \\
& \Phi=L \exp [\mu(x+2 v y)] \exp \left[i v x-i\left(\mu^{2}-v^{2}\right) y\right],
\end{aligned}
$$

where

$$
K=\frac{\kappa}{2 \mu} \sum_{m, n=1}^{N} \frac{\bar{q}_{m} q_{n}}{\varrho_{m}^{3}+\bar{\varrho}_{n}^{3}}, \quad L=-\sum_{m=1}^{N} q_{m} .
$$

If all the quantities $\varrho_{m}^{3}$ are taken different, then it follows from inequality (1.32) that the square form $K$ is positive definite. This means that $K>0$ at any real value of the time $t$. Thus, by virtue of (1.6) our solution of the system (1) really has the form of (9) if one puts

$$
f=-\frac{1}{2 \mu} \ln K, \quad A=\frac{1}{2} L K^{-1 / 2} .
$$


Taking account of (1.31) we get that the quantities $K$ and $L$ determined above satisfy the relation

$$
\mu \frac{d K}{d t}+8\left(\mu^{2}-3 v^{2}\right) \mu^{2} K+2 \kappa|L|^{2}=0 .
$$

Hence it follows that the quantities $f$ and $A$ determined by (1.33) and (1.34) satisfy relation (10). Then, the equality

$$
\frac{\partial^{2} f}{\partial t^{2}}=\frac{\kappa}{\mu^{2} K^{2}}\left(K \frac{d}{d t}|L|^{2}-|L|^{2} \frac{d K}{d t}\right)
$$

is valid. According to (1.33) it follows from this equality that if among the quantities $\varrho_{m}^{3}$ there are at least two different ones, then the inequality $\frac{d^{2} f}{d t^{2}} \neq 0$ is valid, i.e., the phase velocity of the wave (9) in this case is not constant in time.

The considered solitary wave does not exhaust all possible cases. To verify this we put

$$
\begin{aligned}
q_{m}= & \sum_{r=0}^{\infty} a_{m, r} \exp \left[-4\left(\omega_{m}^{3}+\bar{\varrho}_{m, r}^{3}\right) t\right], \quad m=1, \ldots, N, \\
Q_{m, n}= & C_{m, n} \exp \left[-4\left(\bar{\omega}_{m}^{3}+\omega_{n}^{3}\right) t\right] \\
& +\kappa \sum_{r, s=0}^{\infty} \frac{\bar{a}_{m, r} a_{n, s}}{\varrho_{m, r}^{3}+\varrho_{n, s}^{3}} \exp \left[-4\left(\bar{\omega}_{m}^{3}+\omega_{n}^{3}+\varrho_{m, r}^{3}+\bar{\varrho}_{n, s}^{3}\right) t\right], m, n=1, \ldots, N,
\end{aligned}
$$

where $a_{m, r}, C_{m, n}, \omega_{m}$ and $\varrho_{m, r}$ are the complex parameters. One can easily see that the vector $q$ and matrix $Q$ thus defined satisfy relation (1.3). Moreover, the matrix $Q$ will obviously be Hermitian if the quantities $C_{m, n}$ form the Hermitian matrix. Then, one can easily be convinced that if $C_{m, n}=0$ at $m, n=1, \ldots, N$ and among the quantities $a_{m, r}$ there is only a finite number of nonzero quantities, then the solution of the system (1) obtained by (1.35) describes the interaction of $N$ solitary waves of the form of (9), each of these waves can be obtained from the multi-soliton solution of the system (1) in the way described above, and consequently, the solution itself can be obtained from the multi-soliton solution of the system (1). However, in the case when among the quantities $a_{m, r}$ there is an infinite number of nonzero quantities, the solution of the system (1) obtained by (1.35) cannot be obtained from the multi-soliton solution of this system. There are other possible ways of choosing the vector $q$ and matrix $Q$ which satisfy relation (1.3) and allow one to get other types of solitary waves of the form of (9) differing from the multisoliton ones. Especially, we should like to mention solitary waves of the form of (9) with limited functions $f_{m}$ at all $t \in(-\infty, \infty)$ such that $\frac{d f_{m}}{d t} \neq 0$. Solitary waves of that type make finite motions, i.e., each moves all the time in the finite limits. In the case when the parameters $v_{m}$ of all the waves of the form of (9) participating in the interaction coincide, $m=1, \ldots, N$, these waves form one solitary wave that can decay into several solitary waves of the form (9). In the next paragraph we shall discuss this phenomenon in more detail. 


\section{Formation and Decay of a Bound State in the System (1)}

Now we use the components $q_{m}$ of the vector $q$ and the elements $Q_{m, n}$ of the matrix $Q$ in the following form:

$$
\begin{aligned}
q_{m}= & a_{m} \exp \left[-4\left(\omega_{m}^{3}+\bar{\varrho}_{m}^{3}\right) t\right]+b_{m} \exp \left(4 i \gamma_{m} t\right), \quad m=1, \ldots, N, \\
Q_{m, n}= & \kappa \frac{\bar{a}_{m} a_{n}}{\varrho_{m}^{3}+\bar{\varrho}_{n}^{3}} \exp \left[-4\left(\bar{\omega}_{m}^{3}+\omega_{n}^{3}+\varrho_{m}^{3}+\bar{\varrho}_{n}^{3}\right) t\right] \\
& -\kappa \frac{\bar{a}_{m} b_{n}}{\omega_{n}^{3}-\varrho_{m}^{3}+i \gamma_{n}} \exp \left[-4\left(\bar{\omega}_{m}^{3}+\varrho_{m}^{3}-i \gamma_{n}\right) t\right] \\
& -\kappa \frac{a_{n} \bar{b}_{m}}{\bar{\omega}_{m}^{3}-\bar{\varrho}_{n}^{3}-i \gamma_{m}} \exp \left[-4\left(\omega_{n}^{3}+\bar{\varrho}_{n}^{3}+i \gamma_{m}\right) t\right] \\
& -\kappa \frac{\bar{b}_{m} b_{n}}{\bar{\omega}_{m}^{3}+\omega_{n}^{3}-i\left(\gamma_{m}-\gamma_{n}\right)} \exp \left[-4 i\left(\gamma_{m}-\gamma_{n}\right) t\right], m, n=1, \ldots, N,
\end{aligned}
$$

where $a_{m} \neq 0, b_{m} \neq 0, \omega_{m}$ and $\varrho_{m}^{3}$ are the complex parameters and $\gamma_{m}$ are the real parameters. It is easy to verify that the vector $q$ and matrix $Q$ thus determined satisfy relation (1.3). Then, assume

$$
\omega_{m}=\mu_{m}+i v, \varrho_{m}^{3}=-\bar{\omega}_{m}^{3}+\frac{1}{4}\left(\mu_{m} \tau_{m}+i \sigma_{m}\right), \quad m=1, \ldots, N,
$$

where $\mu_{m}, \sigma_{m}, \tau_{m}$ and $v$ are the real parameters satisfying the inequalities

$$
\kappa \mu_{m}<0, \quad \mu_{m} \tau_{m}<0,\left(\mu_{m}^{2}-3 v^{2}\right) \mu_{m}>0, \quad m=1, \ldots, N .
$$

In this case, the elements $Q_{m, n}$ of the matrix $Q$ determined by equalities (2.1) admit the representation

$$
\begin{aligned}
Q_{m, n}= & -4 \kappa \exp \left[-4\left(\bar{\omega}_{m}^{3}+\omega_{n}^{3}\right) t\right] \\
& \times \int_{-\infty}^{t} \bar{q}_{m}\left(t^{\prime}\right) q_{n}\left(t^{\prime}\right) \exp \left[4\left(\bar{\omega}_{m}^{3}+\omega_{n}^{3}\right) t^{\prime}\right] d t^{\prime} .
\end{aligned}
$$

Hence, it follows that the Hermitian matrix $\kappa Q$ will be nonpositive. Then, it follows from the condition $\kappa \mu_{m}<0, m=1, \ldots, N$, that the Hermitian matrix $\kappa P$ determined by (1.1) and (1.2) will be nonpositive as well. Consequently, by virtue of (1.5) at any real $x, y$ and $t$ the inequality $D \geqq 1$ is valid. Thus, the solution of the system (1) determined by expressions (2.1) according to (1.6) has no singularities at any real values of $x, y$ and $t$.

Let us now analyse the dynamics of this solution. For this purpose we use the matrix $G$ with the elements $G_{m, n}$ of the form

$$
\begin{aligned}
G_{m, n}= & \frac{\bar{b}_{m} b_{n}}{\bar{\omega}_{m}^{3}+\omega_{n}^{3}-i\left(\gamma_{m}-\gamma_{n}\right)} \\
& \cdot \exp \left[-4 i\left(\gamma_{m}-\gamma_{n}\right) t\right], \quad m, n=1, \ldots, N .
\end{aligned}
$$

If the quantities $\hat{\omega}_{m}^{3}=\omega_{m}^{3}+i \gamma_{m}$ are all different, $m=1, \ldots, N$, then the Hermitian matrix $G$ on the basis of (2.2) and (2.3) will be positive definite. Further, taking account of (2.1)-(2.3) we have $Q_{m, n}+\kappa G_{m, n} \rightarrow 0$ as $t \rightarrow-\infty, m, n=1, \ldots, N$. 
Hence, it follows that there exist such a constant $c_{0}>0$ and moment of time $t_{0}$ that at all $t \leqq t_{0}$ the inequality $(-\kappa)^{N} \operatorname{det} Q \geqq c_{0}$ holds. Finally, if the quantities $\mu_{m}$ are all different, $m=1, \ldots, N$, then according to (1.1) and (1.2) we have $\operatorname{det} P \neq 0$. Assume now $z=(x+2 v y) \kappa$. Then, as $z \rightarrow \infty$ according to (1.1), (1.2) and (2.3) we get that $D \rightarrow 1$ and $\Phi \rightarrow 0$ uniformly in $t \in\left(-\infty, t_{0}\right]$, i.e., $u \rightarrow 0$ and $\varphi \rightarrow 0$ as $z \rightarrow \infty$ uniformly in $t \in\left(-\infty, t_{0}\right]$. On the other hand, using (2.3) we can verify that uniformly in $t \in\left(-\infty, t_{0}\right]$ as $z \rightarrow-\infty$, the following relations are valid:

$$
D \exp \left(-2 \kappa \mu_{0} z\right) \rightarrow 2^{-N} \omega_{0} \operatorname{det} Q, \quad \Phi D^{-1} \rightarrow 0,
$$

where

$$
\mu_{0}=\sum_{m=1}^{N} \mu_{m}, \quad \omega_{0}=\prod_{m=1}^{N} \mu_{m}^{-1} \cdot \prod_{1 \leqq m<n \leqq N}\left(\frac{\mu_{m}-\mu_{n}}{\mu_{m}+\mu_{n}}\right)^{2} .
$$

Hence, it follows that as $z \rightarrow-\infty$ we have $u \rightarrow 0$ and $\varphi \rightarrow 0$ uniformly in $t \in\left(-\infty, t_{0}\right]$. Thus, at $t \in\left(-\infty, t_{0}\right]$ our solution of the system (1) really describes the solitary wave executing a finite motion, i. e., for all $t \in\left(-\infty, t_{0}\right]$ it is in a certain band of the form $|x+2 v y| \leqq C_{0}$, where $C_{0}>0$.

Now let us see what will happen to our solution as $t \rightarrow \infty$. For this purpose we assume the quantities $\tau_{m}$ entering into (2.2) to be all different, $m=1, \ldots, N$, and their numeration is such that at any $m \neq n$ the inequality

$$
\left(\tau_{m}-\tau_{n}\right) \mu_{m}>0 \quad \text { if } m<n
$$

holds. Further, we take the diagonal matrix $M$ of the form

$$
M=\exp (-\mu \tau t)
$$

where $\tau$ is the real parameter, and $\mu$ is the diagonal matrix with the elements $\mu_{m}$ on the principal diagonal, $m=1, \ldots, N$, i.e.,

$$
\mu=\operatorname{diag}\left(\mu_{1}, \ldots, \mu_{N}\right) .
$$

By virtue of (1.5) the equalities

$$
D=\operatorname{det}\left|\begin{array}{rr}
\mathbb{1} & -\hat{Q} \\
\hat{P} & \mathbb{1}
\end{array}\right|, \quad \Phi=\operatorname{det}\left|\begin{array}{rrr}
0 & 0 & \tilde{\eta} \\
0 & \mathbb{1} & -\hat{Q} \\
\zeta & \hat{P} & \mathbb{1}
\end{array}\right|
$$

are valid where

$$
\hat{P}=M P M, \quad \hat{Q}=M^{-1} Q M^{-1}, \quad \zeta=M \lambda, \quad \eta=M^{-1} q .
$$

Based on (1.1), (1.2), (2.5) and (2.8) the elements $\hat{P}_{m, n}$ of the matrix $\hat{P}$ have the form

$$
\hat{P}_{m, n}=\frac{\zeta_{m} \bar{\zeta}_{n}}{\omega_{m}+\bar{\omega}_{n}}, \quad m, n=1, \ldots, N
$$

where $\zeta_{1}, \ldots, \zeta_{N}$ are the components of the vector-column $\zeta=M \lambda$. According to (1.1), (2.2), (2.5), and (2.6) the equality

$$
\zeta_{m}=\exp \left(\mu_{m} z\right) \exp \left[i v x-i\left(\mu_{m}^{2}-v^{2}\right) y\right], \quad m=1, \ldots, N,
$$


is valid, where $z=x+2 v y-\tau t$. Finally, taking account of (2.1), (2.2), (2.5), (2.6), and (2.8) we get that the components $\eta_{1}, \ldots, \eta_{N}$ of the vector-column $\eta=M^{-1} q$ and the elements $\hat{Q}_{m, n}$ of the matrix $\hat{Q}$ can be written as follows:

$$
\begin{aligned}
& \eta_{m}= \exp \left[\mu_{m}\left(\tau-\tau_{m}\right) t\right]\left\{a_{m} \exp \left(i \sigma_{m} t\right)\right. \\
&\left.+b_{m} \exp \left[\left(\mu_{m} \tau_{m}+4 i \gamma_{m}\right) t\right]\right\}, \quad m=1, \ldots, N \\
& \hat{Q}_{m, n}= \exp \left[\mu_{m}\left(\tau-\tau_{m}\right) t+\mu_{n}\left(\tau-\tau_{n}\right) t\right] \\
& \times\left\{\kappa \frac{\bar{a}_{m} a_{n}}{\varrho_{m}^{3}+\bar{\varrho}_{n}^{3}} \exp \left[-i\left(\sigma_{m}-\sigma_{n}\right) t\right]\right. \\
&-\kappa \frac{\bar{a}_{m} b_{n}}{\omega_{n}^{3}-\varrho_{m}^{3}+i \gamma_{n}} \exp \left[\mu_{n} \tau_{n} t-i\left(\sigma_{m}-4 \gamma_{n}\right) t\right] \\
&-\kappa \frac{a_{n} \bar{b}_{m}}{\bar{\omega}_{m}^{3}-\bar{\varrho}_{n}^{3}-i \gamma_{m}} \exp \left[\mu_{m} \tau_{m} t+i\left(\sigma_{n}-4 \gamma_{m}\right) t\right] \\
&\left.-\kappa \frac{\bar{b}_{m} b_{n}}{\bar{\omega}_{m}^{3}+\omega_{n}^{3}-i\left(\gamma_{m}-\gamma_{n}\right)} \exp \left[\left(\mu_{m} \tau_{m}+\mu_{n} \tau_{n}\right) t-4 i\left(\gamma_{m}-\gamma_{n}\right) t\right]\right\} \\
& \quad m, n=1, \ldots, N .
\end{aligned}
$$

Now assume that the parameter $\tau \in(-\infty, \infty)$ entering into $(2.5)$ and $(2.11)$ is chosen so that at any $m=1, \ldots, N$ the inequality

$$
\left(\tau-\tau_{m}\right) \mu_{m}<0, \quad m=1, \ldots, N,
$$

is fulfilled. Then, according to (2.3) and (2.11) we get that as $t \rightarrow \infty$ the relations

$$
\eta_{m} \rightarrow 0, \quad m=1, \ldots, N, \quad \hat{Q}_{m, n} \rightarrow 0, \quad m, n=1, \ldots, N,
$$

are valid. Hence, by virtue of (2.7), (2.9) and (2.10) it follows that at any fixed $z=x+2 v y-\tau t$ and $t \rightarrow \infty$ we have $D \rightarrow 1, \Phi \rightarrow 0$, i.e., based on (1.6) at any fixed $z$ and as $t \rightarrow \infty$ we get that $u \rightarrow 0$ and $\varphi \rightarrow 0$. This means that as $t \rightarrow \infty$ our solution has no moving waves with the phase velocity $\tau$ satisfying the condition (2.12). On the other hand, assume that the parameter $\tau \in(-\infty, \infty)$ is chosen so that at any $m=1, \ldots, N$ the inequality

$$
\left(\tau-\tau_{m}\right) \mu_{m}>0, \quad m=1, \ldots, N,
$$

is valid. With (2.3) and (2.11) we get that in this case as $t \rightarrow \infty$ the following asymptotics hold:

$$
\begin{aligned}
& \eta_{m} \exp \left[\mu_{m}\left(\tau_{m}-\tau\right) t\right] \sim a_{m} \exp \left(i \sigma_{m} t\right), \quad m=1, \ldots, N, \\
& \hat{Q}_{m, n} \exp \left[\mu_{m}\left(\tau_{m}-\tau\right) t+\mu_{n}\left(\tau_{n}-\tau\right) t\right] \sim \kappa \frac{\bar{a}_{m} a_{n}}{\varrho_{m}^{3}+\bar{\varrho}_{n}^{3}} \\
& \quad \cdot \exp \left[-i\left(\sigma_{m}-\sigma_{n}\right) t\right], \quad m, n=1, \ldots, N .
\end{aligned}
$$


According to (2.7), (2.9), and (2.10) it follows that at any fixed $z$ and $t \rightarrow \infty$ the relations

$$
\begin{aligned}
& D \exp \left[-2 \sum_{m=1}^{N} \mu_{m}\left(\tau-\tau_{m}\right) t\right] \rightarrow(\operatorname{det} \hat{P})(\operatorname{det} R), \\
& \Phi \exp \left[-2 \sum_{m=1}^{N} \mu_{m}\left(\tau-\tau_{m}\right) t\right] \rightarrow 0
\end{aligned}
$$

are fulfilled, where $R$ is the square matrix of an order of $N$ with the elements $R_{m, n}$ of the form

$$
R_{m, n}=\kappa \frac{\bar{a}_{m} a_{n}}{\varrho_{m}^{3}+\bar{\varrho}_{n}^{3}} \exp \left[-i\left(\sigma_{m}-\sigma_{n}\right) t\right], \quad m, n=1, \ldots, N
$$

When the quantities $\varrho_{m}^{3}$ are all different, $m=1, \ldots, N$, it is obvious that $\operatorname{det} R \neq 0$. In this case, by using (1.6) one can easily get that at any fixed $z$ and as $t \rightarrow \infty$ the asymptotics $u \rightarrow 0$ and $\varphi \rightarrow 0$ hold, i.e., our solution of the system (1) as $t \rightarrow \infty$ also does not contain moving waves with the phase velocity $\tau$ satisfying the condition (2.13).

Consider then the case when the parameter $\tau \in(-\infty, \infty)$ is chosen so that at a certain integer $r$ satisfying the condition $1 \leqq r<N$ the inequality

$\left(\tau-\tau_{r}\right) \mu_{r}<0, \quad\left(\tau-\tau_{r+1}\right) \mu_{r+1}>0$

is valid. Hence, according to (2.4) the inequalities

$$
\begin{aligned}
\left(\tau-\tau_{m}\right) \mu_{m}<0 \text { if } m & =1, \ldots, r, \\
& \left(\tau-\tau_{m}\right) \mu_{m}>0 \text { if } m=r+1, \ldots, N
\end{aligned}
$$

are valid. By virtue of (2.3), (2.11), and (2.15) we find that as $t \rightarrow \infty$ the following asymptotics hold:

$$
\begin{aligned}
& \eta_{m} \rightarrow 0 \text { if } m=1, \ldots, r, \\
& \eta_{m} \exp \left[\mu_{m}\left(\tau_{m}-\tau\right) t\right] \sim a_{m} \exp \left(i \sigma_{m} t\right) \text { if } m=r+1, \ldots, N, \\
& \hat{Q}_{m, n} \rightarrow 0 \text { if } m, n=1, \ldots, r, \\
& \hat{Q}_{m, n} \exp \left[\mu_{n}\left(\tau_{n}-\tau\right) t\right] \rightarrow 0 \text { if } m=1, \ldots, r \text { and } n=r+1, \ldots, N, \\
& \hat{Q}_{m, n} \exp \left[\mu_{m}\left(\tau_{m}-\tau\right) t\right] \rightarrow 0 \text { if } m=r+1, \ldots, N \text { and } n=1, \ldots, r, \\
& \hat{Q}_{m, n} \exp \left[\mu_{m}\left(\tau_{m}-\tau\right) t+\mu_{n}\left(\tau_{n}-\tau\right) t\right] \\
& \sim \kappa \frac{\bar{a}_{m} a_{n}}{\varrho_{m}^{3}+\bar{\varrho}_{n}^{3}} \exp \left[-i\left(\sigma_{m}-\sigma_{n}\right) t\right] \text { if } m, n=r+1, \ldots, N .
\end{aligned}
$$

Based on (2.7), (2.9) and (2.10) it follows that at any fixed $z$ and as $t \rightarrow \infty$ the relations

$$
\begin{aligned}
& D \exp \left[-2 \sum_{m=r+1}^{N} \mu_{m}\left(\tau-\tau_{m}\right) t\right] \rightarrow\left(\operatorname{det} P_{r}\right)\left(\operatorname{det} R_{r}\right), \\
& \Phi \exp \left[-2 \sum_{m=r+1}^{N} \mu_{m}\left(\tau-\tau_{m}\right) t\right] \rightarrow 0
\end{aligned}
$$


hold, where $P_{r}$ and $R_{r}$ are the square matrices of an order of $N-r$, respectively, with the elements $P_{r, m, n}$ and $R_{r, m, n}$ of the form

$$
\begin{aligned}
P_{r, m, n} & =\frac{\zeta_{r+m} \bar{\zeta}_{r+n}}{\omega_{r+m}+\bar{\omega}_{r+n}}, \quad m, n=1, \ldots, N-r \\
R_{r, m, n} & =\kappa \frac{\bar{a}_{r+m} a_{r+n}}{\varrho_{r+m}^{3}+\bar{\varrho}_{r+n}^{3}} \exp \left[-i\left(\sigma_{r+m}-\sigma_{r+n}\right) t\right] . \quad m, n=1, \ldots, N-r .
\end{aligned}
$$

Using equalities (1.6) and relations (2.16) we easily find that at any fixed $z$ and as $t \rightarrow \infty$ the asymptotics $u \rightarrow 0$ and $\varphi \rightarrow 0$ hold, i.e., our solution as $t \rightarrow \infty$ has no moving waves with the phase velocity $\tau$ satisfying the condition (2.14). Thus, from the afore-said we get that by virtue of (2.12)-(2.14) our solution of the system (1) as $t \rightarrow \infty$ has no moving waves with the phase velocity $\tau \neq \tau_{m}, m=1, \ldots, N$.

Finally, consider the case when $\tau=\tau_{r}, r=1, \ldots, N$. According to (2.3), (2.4), (2.11) and equality $\tau=\tau_{r}$ we get that in this case as $t \rightarrow \infty$ the following asymptotics hold:

$$
\begin{aligned}
& \eta_{m} \rightarrow 0 \quad \text { if } m=1, \ldots, r-1, \\
& \eta_{m} \exp \left[\mu_{m}\left(\tau_{m}-\tau_{r}\right) t\right] \sim a_{m} \exp \left(i \sigma_{m} t\right) \quad \text { if } m=r, \ldots, N, \\
& \hat{Q}_{m, n} \rightarrow 0 \quad \text { if } m, n=1, \ldots, r-1, \\
& \hat{Q}_{m, n} \exp \left[\mu_{n}\left(\tau_{n}-\tau_{r}\right) t\right] \rightarrow 0 \quad \text { if } m=1, \ldots, r-1 \text { and } n=r, \ldots, N, \\
& \hat{Q}_{m, n} \exp \left[\mu_{m}\left(\tau_{m}-\tau_{r}\right) t\right] \rightarrow 0 \quad \text { if } m=r, \ldots, N \text { and } n=1, \ldots, r-1, \\
& \hat{Q}_{m, n} \exp \left[\mu_{m}\left(\tau_{m}-\tau_{r}\right) t+\mu_{n}\left(\tau_{n} \sim \tau_{r}\right) t\right] \\
& \sim \kappa \frac{\bar{a}_{m} a_{n}}{\varrho_{m}^{3}+\bar{\varrho}_{n}^{3}} \exp \left[-i\left(\sigma_{m}-\sigma_{n}\right) t\right] \quad \text { if } m, n=r, \ldots, N .
\end{aligned}
$$

According to (2.7), (2.9), and (2.10) it follows that at any fixed $z=x+2 v y-\tau_{r} t$ and $t \rightarrow \infty$ the relations

$$
\begin{aligned}
& D \exp \left[-2 \sum_{m=r}^{N} \mu_{m}\left(\tau_{r}-\tau_{m}\right) t\right] \rightarrow\left(\operatorname{det} P_{r-1}\right)\left(\operatorname{det} R_{r-1}\right)+\left(\operatorname{det} P_{r}\right)\left(\operatorname{det} R_{r}\right) \\
& \Phi \exp \left[-2 \sum_{m=r}^{N} \mu_{m}\left(\tau_{r}-\tau_{m}\right) t\right] \rightarrow-\left(\operatorname{det} \hat{P}_{r-1}\right)\left(\operatorname{det} \hat{R}_{r-1}\right)
\end{aligned}
$$

are valid. Here, the matrices $P_{r}$ and $R_{r}$ at $r=0,1, \ldots, N-1$ are the square matrices of an order of $N-r$ with the elements $P_{r, m, n}$ and $R_{r, m, n}$ determined by expressions (2.17) and at $r=N$ we assume by definition $\operatorname{det} P_{N}=\operatorname{det} R_{N}=1$. Further, the matrix $\hat{P}_{r}$ is obtained from the matrix $P_{r}$ by substituting the elements of the first column of this matrix by the quantities $\zeta_{r+1}, \ldots, \zeta_{N}, r=0,1, \ldots, N-1$, and the matrix $\hat{R}_{r}$ is obtained from the matrix $R_{r}$ by substituting the first row of this matrix by the quantities $a_{r+1} \exp \left(i \sigma_{r+1} t\right), \ldots, a_{N} \exp \left(i \sigma_{N} t\right), r=0,1, \ldots, N-1$. Taking 
account of (2.9), (2.10) and (2.17) it follows from relation (2.18) that as $t \rightarrow \infty$ our solution contains $N$ solitary waves of the form

$$
\begin{aligned}
& u=\frac{2 \mu_{r}^{2}}{\cosh ^{2}\left[\mu_{r}\left(x+2 v y-\tau_{r} t\right)+f_{r}\right]}, \\
& \varphi=A_{r} \frac{\exp \left[i v x-i\left(\mu_{r}^{2}-v^{2}\right) y\right]}{\cosh \left[\mu_{r}\left(x+2 v y-\tau_{r} t\right)+f_{r}\right]},
\end{aligned}
$$

where $f_{r}$ and $A_{r}$ are the limited functions $t \in(-\infty, \infty)$. Thus, our solution really describes the decay of one solitary wave into $N$ solitary waves of the form (2.19).

It should be noted that the solution considered above conserves its dynamics under the change of the vector $q$ with the components $q_{m}$ of the form (2.1) by the vector $q^{\prime}$ with the components $q_{m}^{\prime}=q_{m}+\varepsilon g_{m}$, where $\varepsilon$ is the small parameter and $g_{m}$ are arbitrary limited functions $t \in(-\infty, \infty)$. In this case, the elements $Q_{m, n}$ determined by (2.1) of the matrix $Q$ should necessarily be substituted by the quantities $Q_{m, n}^{\prime}$ of the form

$$
\begin{aligned}
Q_{m, n}^{\prime}= & -4 \kappa \exp \left[-4\left(\bar{\omega}_{m}^{3}+\omega_{n}^{3}\right) t\right] \\
& \times \int_{-\infty}^{t} \bar{q}_{m}^{\prime}\left(t^{\prime}\right) q_{n}^{\prime}\left(t^{\prime}\right) \exp \left[4\left(\bar{\omega}_{m}^{3}+\omega_{n}^{3}\right) t^{\prime}\right] d t^{\prime} .
\end{aligned}
$$

In conclusion, it should be emphasized that the above-described process of decaying one solitary wave into an arbitrary number of solitary waves does not exhaust all possibilities of the obtained class of solutions of the system (1).

\section{References}

1. Mel'nikov, V.K.: On equations for wave interactions. Lett. Math. Phys. 7, 129 (1983)

2. Zakharov, V.E., Kuznetsov, E.A.: Multi-scale expansions in the theory of systems integrable by inverse scattering transform. Physica 18D, 455 (1986)

3. Mel'nikov, V.K.: A direct method for deriving a multi-soliton solution for the problem of interaction of waves on the $x, y$ plane. Commun. Math. Phys. 112, 639 (1987)

4. Mel'nikov, V.K.: Capture and confinement of solitons in nonlinear integrable systems. Commun. Math. Phys. 120, 451 (1989)

5. Satsuma, Y.: $N$-soliton solution of the two-dimensional Korteweg-de Vries equation. J. Phys. Soc. Jpn. 40, 286 (1976)

6. Kadomtsev, B. B., Petviashvili, V.I.: Stability of solitary waves in weakly dispersing mediums. Dokl. Akad. Nauk SSSR 192, 753 (1970)

Communicated by H. Araki

Received April 1, 1989 
